\title{
An 88.8-kb Novel Deletion of 19q13.2 \\ Encompassing the ATP1A3 Gene Detected by Array CGH in a Patient with Delayed Psychomotor Development, Generalized Hypotonia and Macrocephaly
}

\author{
Elena García-Payá María Gutiérrez-Agulló Francisco F. García-Prieto \\ Jorge Francés Ferre \\ Laboratory of Molecular Cytogenetics, Instituto de Investigación Sanitaria y Biomédica de Alicante (ISABIAL), \\ Hospital General Universitario de Alicante, Alicante, Spain
}

\section{Keywords}

Array CGH · 19q13.2 - Deletion · Copy number variation · ATP1A3

\begin{abstract}
Many neurodevelopmental disorders are caused by the presence of CNVs. Chromosome microarray technology is widely used to accurately detect CNVs. We report the case of a male, aged 3 years, presenting with delayed psychomotor development, generalized hypotonia, encephalopathy, delayed myelination in the central nervous system, and poor motor coordination. The array $\mathrm{CGH}$ revealed an interstitial deletion of chromosome 19q13.2 with a size of $88.8 \mathrm{~kb}$ involving 3 OMIM genes: RABAC1, ARHGEF1, and ATP1A3. Heterozygous mutations in the ATP1A3 gene are associated with delayed psychomotor development, alternating hemiplegia of childhood type 2 (AHC2), dystonia type 12, and cerebellarataxia-areflexia-pes cavus-optic atrophy-sensorineural hearing loss syndrome, also called CAPOS syndrome. The phenotypic expression of partial ATP1A3 deletion is, however, poorly described in the literature. The deletion was
\end{abstract}

confirmed by MLPA, and we identified a hitherto undescribed novel deletion of exons $3 b-21$ of the ATP1A3 gene. Our data suggest that the deletion of the ATP1A3 gene is a causative factor of the AHC2 phenotype in the patient.

(c) 2021 S. Karger AG, Basel

\section{Introduction}

Neurodevelopmental disorders (NDD) are of major interest to the national health systems of developed countries: in the US, a recent study estimated that the prevalence of NDD was 4.7\% [Zablotsky et al., 2019] and similar percentages have been found for Spain [Carballal Mariño et al., 2018]. These disorders present a variety of manifestations, e.g., intellectual disability (ID), neurodevelopmental delay, motor delay, and autistic behavior. Many of these medical conditions and diseases involve $\mathrm{CNVs}$ in the genome. Today, the detection of CNVs by chromosomal microarray is the first-tier diagnostic test for patients with ID, autistic behavior, and/or congenital anomalies [Miller et al., 2010]. A systematic review and 
meta-analysis by Sagoo et al. [2009] established a diagnostic yield of causal genetic abnormalities at $10 \%$. In a more recent study, Di Gregorio et al. [2017] revealed the detection of non-benign CNVs in $29 \%$ of the consecutive cases with ID/NDD.

In the present study, we describe an interstitial deletion of $88.8 \mathrm{~kb}$ in $19 \mathrm{q} 13.2$ found in a male patient diagnosed with NDD, delayed psychomotor development, generalized hypotonia, and poor motor coordination. The detected alteration in our patient affects 3 OMIM genes: RABAC1, ARHGEF1, and ATP1A3. A single OMIM morbid gene, the ATP1A3 gene $\left({ }^{*} 182350\right)$ is partially deleted. Heterozygous mutations in this gene lead to 3 mental/neurological disorders that share some clinical features: alternating hemiplegia of childhood type 2 (AHC2; OMIM \#614820), cerebellar ataxia-areflexia-pes cavus-optic atrophy-sensorineural hearing loss (CAPOS; OMIM \#601338) and dystonia type 12 (DYT12; OMIM $\# 128235$ ). No deletions such as the one detected in the patient's array have been described to date. In addition, cases of deletions affecting the ATP1A3 gene are rare, and their clinical impact is unknown.

\section{Materials and Methods}

\section{Clinical Report}

The proband is the second son of healthy, nonconsanguineous parents with no significant family history. He was born at full term $(39+5$ weeks of gestation), via emergency caesarean section due to breech presentation. He was born with a normal weight and height, but he presented with macrocephaly. At $10 \mathrm{~h}$ of life, he showed neonatal respiratory distress requiring intensive care, which was later resolved without any further complications. Generalized hypotonia was observed from the time of birth, and it persisted at the cervical level until the age of 2 months. An electroencephalogram (EEG) study was performed and severe encephalopathy with a burst-suppression was reported, highly suggestive of a congenital metabolic condition. In addition, the patient had an interictal EEG abnormality, with multifocal independent epileptiform discharges predominantly in the right cerebral hemisphere. Magnetic resonance imaging (MRI) showed a delayed myelination of white matter. An electromyography study showed a mild to moderate diffuse myopathy without any signs of inflammation or necrosis. The brain ultrasound was normal for his age. Several biochemical tests were conducted, including screening for mucopolysaccharide and congenital metabolic disorders, without informative results. Initial molecular studies included a peripheral blood chromosome analysis with a normal male karyotype $(46, \mathrm{XY})$ and a negative Prader-Willi syndrome genetic test. A further EEG was performed at 3 months of age showing nonspecific mild encephalopathy with discrete slow wave activity, with no apparent signs of epilepsy or interhemispheric asymmetry. Vision and hearing examinations were normal.

ATP1A3 Deletion Associated AHC2

Detected by Array
He started to walk at the age of 2 years and 9 months and showed difficulties with fine and gross motor skills. He could only understand simple orders. His vocabulary consisted of a few words with referential meanings and the emission of sounds. Generalized hypotonia and macrocephaly (cranial perimeter of $54.5 \mathrm{~cm},>97 \mathrm{th}$ centile) were persisting at that age. Unusual behavior was observed, with aggressive outbursts, self-injury, social phobia, and low frustration tolerance. He is currently receiving special needs education for children and is incontinent. An autism spectrum disorder and neurodevelopmental delay was suspected and a genomic array was performed.

\section{Genetic Analysis}

DNA was extracted from peripheral blood using MagNA Pure Compact System (Roche Diagnostics GmbH, Germany). A chromosomal microarray was performed on the proband using the whole-genome coverage platform (SurePrint-G3-Human-CGH Microarray-8x60K, design Id021924, Agilent Technologies, Santa Clara, CA, USA). The array was analyzed by using Agilent Cytogenomics 5.0.2.5 software (Agilent Technologies). Cartagenia software (Agilent Technologies) was used to interpret the CNVs. We evaluated the CNVs according to the consensus recommendation of the American College of Medical Genetics and Genomics and the Clinical Genome Resource [Riggs et al., 2020]. The nomenclature of arrays was described in accordance with the International System for Human Cytogenetic Nomenclature [ISCN, 2016] and using the Human Genome build Genome Assembly hg19 (build 38).

The genomic copy number aberration was confirmed by MLPA using a P059 probemix (MRC-Holland, The Netherlands). All assay procedures were carried out in accordance with the manufacturer's protocol. This genetic analysis was derived to another laboratory (Reference Laboratory, Spain). The MLPA fragment analysis data were generated by means of the 3500xL Genetic Analyzer (Applied Biosystems, Waltham, MA, USA). The data were analyzed using the Coffalyser software (MRC-Holland,The Netherlands).

\section{Results}

The array revealed an interstitial pathogenic deletion involving chromosome $19 \mathrm{q} 13.2$ with a size of $88.8 \mathrm{~kb}$, genomic coordinates (42398436_42487224), encompassing 3 OMIM genes (RABAC1, ARHGEF1, and ATP1A3; shown in Fig. 1a). The ARHGEF1 gene showed a full loss while $R A B A C 1$ and $A T P 1 A 3$ showed a probable partial loss. The $\mathrm{CNV}$ was not documented in the ClinGen database nor in the Database of Genomic Variants. In the DECIPHER database, one female patient (Id 249925) with a $431.87-\mathrm{kb}$ de novo heterozygous deletion involving the ATP1A3 gene was found, but no phenotype data were available. The $A T$ $P 1 A 3$ gene pLI predictor score was 1 , indicating that the $A T P 1 A 3$ gene is intolerant to a heterozygous loss of function. Heterozygous mutations of ATP1A3 are known to be associated with AHC2, CAPOS syndrome, and DYT12 with autosomal dominant inheritance. The CNV encompasses ATP1A3 whose function is well-characterized and 


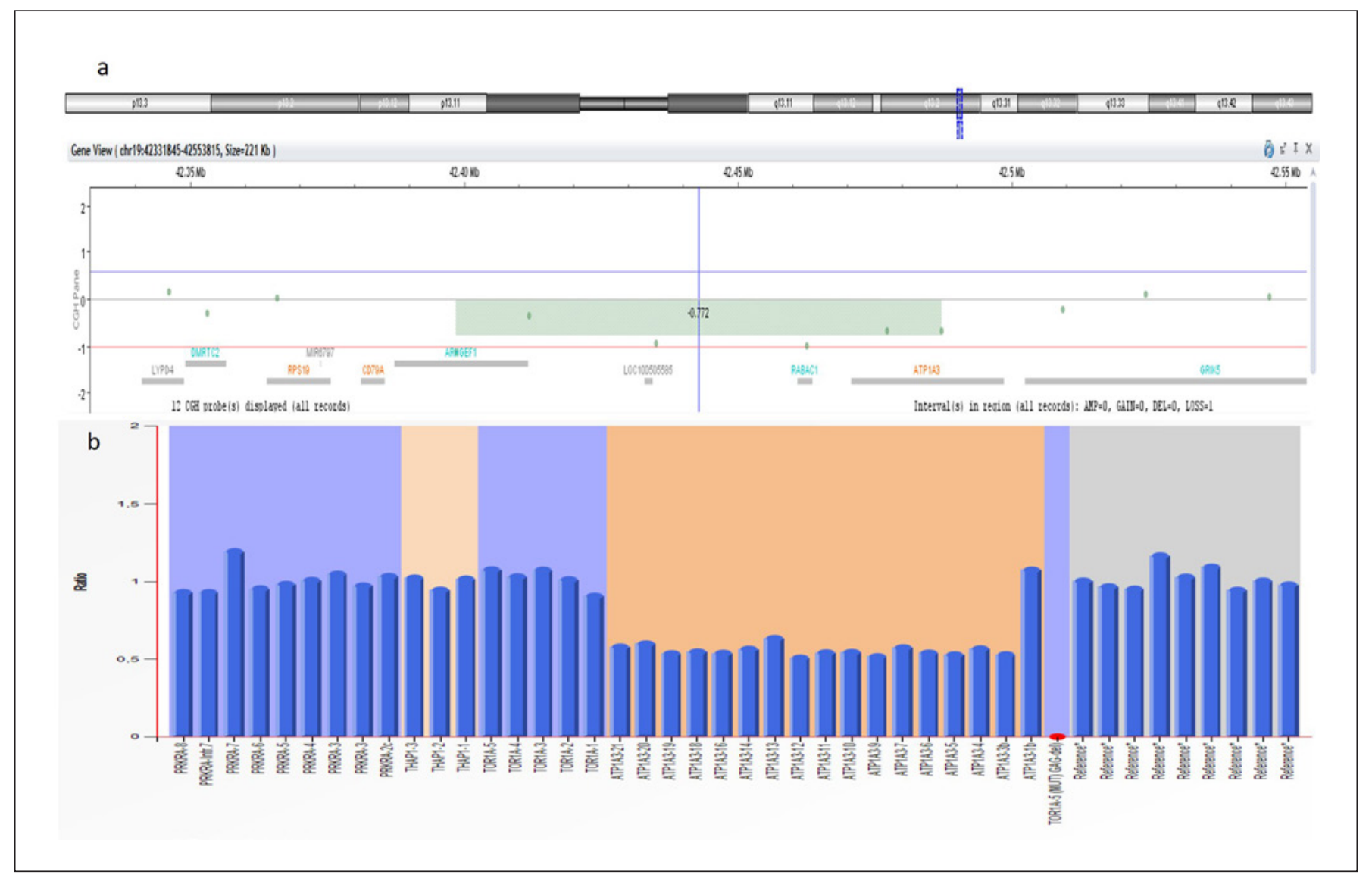

Fig. 1. Genetic analysis. Microarray and MLPA results of the patient with the ATP1A3 deletion. a Microarray profile with a copy number loss at 19q13.2 (42398436_42487224), analysis results viewed using Agilent Cytogenomics software. They show 2 probe deletions involving the ATP1A3 gene (green bar). b The MLPA scatter plot showing the deletion of a probe site on ATP1A3.

is a likely causal factor of the patient's phenotype. The $R A$ $B A C 1$ and $A R H G E F 1$ genes are not related to a pathological phenotype. Except for the 19q13.2 loss, no other CNVs were detected in the patient. As a result, the CNV detected was classified as Class 4 (likely pathogenic) by our laboratory. However, the array results showed only 2 probes in the $-0.5 \log 2$ ratio involving the ATP1A3 gene (shown in Fig. 1a), and a further evaluation was necessary to clarify the CNV's genomic content. We decided to confirm a reduced gene dosage by MLPA.

MLPA results confirmed the genomic copy number aberration in the ATP $1 A 3$ gene. We identified a novel deletion of exons $3 \mathrm{~b}-21$ of the ATP1A3 gene. No probes in the P059 probemix hybridize with the last exons of the ATP1A3 gene, nor with exon 2. Considering this result and the array's genomic coordinates, we concluded that the deletion started between exon 1 and 3 (Fig. 1b) and ended at exon 21 (included).
The patient's parents and his older brother are seemingly unaffected by ATP1A3-related neurological disorders; no analysis was performed. The CNV was considered apparently de novo. A summary of clinical features seen in our patient is shown in Table 1 .

\section{Discussion}

The NDD diagnoses are clinically important for the pediatric population because early assessment can help health workers as well as families to provide efficient care and therapy, and improve their quality of life [Schalock et al., 2002]. Studies and diagnoses remain a clinical challenge due to the wide range of NDD and their clinical manifestations. On the one hand, the diagnosis takes a long time and generally requires costly healthcare resources, such as MRI, electromyography, and genetic 
Table 1. Phenotypic features of our case versus clinical features in a similar case

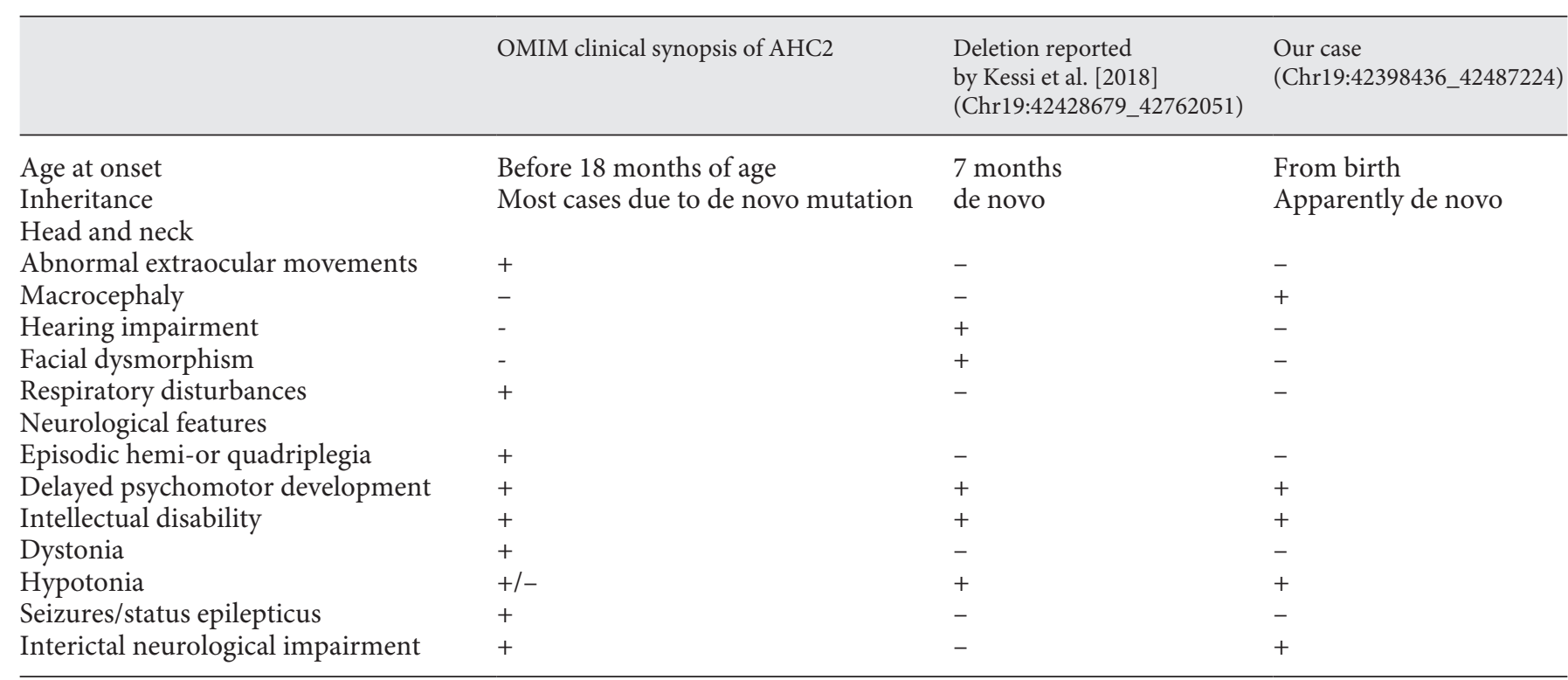

AHC2, alternating hemiplegia of childhood type 2; OMIM, Online Mendelian Inheritance in Man; +, feature usually occurs or presented; -, feature not described or not presented; +/-, feature may occur.

tests [Cohen et al., 2012]. On the other hand, some NDD patients may be left undiagnosed or without a clear diagnosis, despite undergoing all these tests [Leonard and Wen, 2002]. Microarray allows identifying chromosomal anomalies in a more precise, sensitive and accurate way, improving the yield and resolution compared to traditional karyotyping. Nevertheless, chromosomal microarray limitations depend on the length of DNA probes used for covering the genome and the probe spacing. We did in fact detect a novel deletion in our patient that encompassed a single OMIM morbid gene, namely ATP1A3. The array resolution, however, was not sufficiently high to confirm the genomic copy number ATP1A3 aberration. By carrying out an ATP1A3 gene MLPA, we could confirm this copy number aberration and consequently, a genetic diagnosis based on CNV was made. Therefore, once again, the chromosomal array played a key role in the genetic diagnosis of NDD.

ATP1A3 compromises 23 exons and encodes the a3subunit of the sodium-potassium ATPase $\left(\mathrm{Na}^{+} / \mathrm{Ka}^{+}\right.$ATPase). The activity of this ATPase is crucial to transmit the nerve impulses to the synapse and the muscle's motor response [Rodacker et al., 2006; Bøttger et al., 2011]. Heterozygous mutations in $A T P 1 A 3$ have been linked to DYT12, AHC2 and CAPOS syndrome, and some clinical features overlap and resemble each other. Affected patients often present with characteristics of more than one syndrome. Others present with intermediate phenotypes. Others still may exhibit only a few clinical features of 1,2 or 3 syndromes caused by mutations in the ATP1A3 gene. It thus becomes difficult to make a diagnosis [Roubergue et al., 2013; Boelman et al., 2014; Rosewich et al., 2014; Capuano et al., 2020].

DYT12, also known as rapid onset dystonia-parkinsonism, typically begins in late adolescence or early adulthood (in the range of 15-45 years of age). Childhood onset is rare. DYT12 is an autosomal dominant disorder. Our patient's phenotype does not seem to fit the DYT12 phenotype. He presented with some of these symptoms at birth and before 12 months of age. The hypotonia in our patient beginning at birth is not a frequent clinical manifestation of DYT12. One infant aged 4 years was reported with signs of hypotonia at 3 years of age [Anselm et al., 2009] and a DYT12 phenotype. The DYT12 diagnosis is unlikely due to the different age of onset (4 years vs. from birth) and the dominant family pattern. DYT12 is usually a hereditary condition (de novo mutations are not frequent), and our patient's parents seem to be unaffected regarding DYT12 as well as AHC2 and CAPOS.

CAPOS syndrome's main characteristics are summarized by its acronym (cerebellar ataxia-areflexia -pes cavus-optic atrophy-sensorineural hearing loss). CAPOS syndrome has been described in only few individualswho have an apparently de novo pathogenic variant in AT- 
P1A3 [Demos et al., 2014; Duat Rodriguez et al., 2017]. In the case of our patient, we concluded that CAPOS syndrome could be excluded from the differential diagnostics for several reasons: (i) the low probability of CAPOS syndrome because it is a highly uncommon disease with only a few probands reported worldwide; (ii) our patient presents delayed psychomotor development that seems to be uncharacteristic of patients with CAPOS syndrome, and (iii) the absence of pes cavus, sensorineural hearing loss, and optic atrophy in our patient who had normal vision and hearing test results.

AHC2 is a neurological disorder that typically begins before 18 months of age. Its manifestations include, among others, delayed psychomotor development, dystonia, ID, interictal neurological impairment and episodic hemi-or quadriplegia. The pathogenic variant is usually originated de novo. After reviewing the patient's phenotypic spectrum and the clinical characteristics of the 3 disorders caused by mutations in the ATP $1 A 3$ gene, we believe that our patient is closest to the AHC2 phenotype (Table 1). The fact that the patient does not have typical AHC2 clinical characteristics may be due to his age. They may appear at a later stage in childhood.

While most ATP1A3 pathogenic variants identified were single nucleotide substitution, to the best of our knowledge, only one case with a similar deletion has been reported, and very little is known about its phenotype expression [Kessi et al., 2018]. These authors showed a de novo heterozygous deletion of the 19q13.2 chromosome (Chr19:42428679_42762051) in a patient with a similar phenotype, but the deletion was larger ( $333 \mathrm{~kb}$ vs. $88 \mathrm{~kb}$ ). This deletion contained the ATP $1 A 3$ and ERF genes and was classified by this study as rare pathogenic CNV. They consider ATP1A3 as a dosage-sensitive gene and thus, the $\mathrm{CNV}$ would be the principal cause of the phenotypic expression shown in this case. The patient described by Kessi et al. [2018] had a clinical phenotype resembling the one found in our case (Table 1).

It is still complex to determine the significance of CNVs and to interpret them. In the case of our patient's detected chromosomal aberration, we considered the case was likely pathogenic because the deletion involved multiple exons in an established haploinsufficiency gene and because the patient's phenotype is compatible with the clinical manifestations of patients with $A T P 1 A 3$ gene mutations. Nevertheless, additional reports are necessary to completely elucidate the effects of abnormal ATP1A3 CNVs. There is indeed still a limited amount of evidence to support that the ATP1A3 deletion is a causative factor of DYT12, AHC2 or CAPOS syndrome.

\section{Conclusion}

To conclude, we report a novel apparently de novo interstitial heterozygous deletion of 19q13.2 involving the $A T P 1 A 3$ gene in a patient presenting with delayed psychomotor development, generalized hypotonia, and macrocephaly. No deletions such as the one detected have been described to date. We believe that the pathology found in our patient matches to a AHC2 phenotype. The present study contributes to expanding the literature on $A T P 1 A 3$ mutations and to a better understanding of the clinical features associated with ATP1A3 deletion.

\section{Acknowledgement}

We would like to acknowledge the consultants and technical staff of the Clinical Laboratory Department of the Hospital General Universitario de Alicante, Spain. We thank Dr. Blaya for the support and collaboration in this case report. We would also like to thank the patient and his parents for participating in this study and giving their consent.

\section{Statement of Ethics}

The genetic analysis was performed in accordance with the Good Clinical Practice Standards. The patient's parents gave their informed consent before the genetic studies began in compliance with the Helsinki Declaration. The genetic analysis was approved by Ethics Committee of Instituto de Investigación Sanitaria y Biomédica de Alicante (ISABIAL).

\section{Conflict of Interest Statement}

The authors have no conflicts of interest to declare.

\section{Funding Sources}

No funding was obtained for this study.

\section{Author Contributions}

E.G. and F.F. contributed to the conception and design of the study. F.F. contributed to data collection. F.F. and J.F. contributed to genetic analysis. E.G. contributed to data analysis and CNV interpretation. F.F., M.G., and E.G. contributed to writing and editing the manuscript. All authors revised and approved the final version of the manuscript. 


\section{References}

Anselm IA, Sweadner KJ, Gollamudi S, Ozelius LJ, Darras BT. Rapid-onset dystonia-parkinsonism in a child with a novel ATP1A3 gene mutation. Neurology. 2009;73(5):400-1.

Boelman C, Lagman-Bartolome AM, MacGregor DL, McCabe J, Logan WJ, Minassian BA. Identical ATP1A3 mutation causes alternating hemiplegia of childhood and rapid-onset dystonia parkinsonism phenotypes. Pediatr Neurol. 2014;51(6):850-3.

Bøttger P, Tracz Z, Heuck A, Nissen P, RomeroRamos M, Lykke-Hartmann K. Distribution of $\mathrm{Na} / \mathrm{K}$-ATPase alpha 3 isoform, a sodiumpotassium P-type pump associated with rapid-onset of dystonia parkinsonism (RDP) in the adult mouse brain. J Comp Neurol. 2011; 519(2):376-404.

Capuano A, Garone G, Tiralongo G, Graziola F. Alternating hemiplegia of childhood: Understanding the genotype-phenotype relationship of ATP1A3 variations. Appl Clin Genet. 2020;13:71-81.

Carballal Mariño M, Gago Ageitos A, Ares Alvarez J, del Rio Garma M, García Cendón C, Goicoechea Castaño A, et al. Prevalence of neurodevelopmental, behavioural and learning disorders in Pediatric Primary Care (in Spanish). An Pediatr (Engl Ed). 2018;89(3): 153-161.

Cohen E, Berry JG, Camacho X, Anderson G, Wodchis W, Guttmann A. Patterns and costs of health care use of children with medical complexity. Pediatrics. 2012;130(6):e146370.

Demos MK, Van Karnebeek CD, Ross CJ, Adam $\mathrm{S}$, Shen $\mathrm{Y}$, Zhan $\mathrm{SH}$, et al. A novel recurrent mutation in ATP1A3 causes CAPOS syndrome. Orphanet J Rare Dis. 2014;9:15.
Di Gregorio E, Riberi E, Belligni EF, Biamino E, Spielmann M, Ala U, et al. Copy number variants analysis in a cohort of isolated and syndromic developmental delay/intellectual disability reveals novel genomic disorders, position effects and candidate disease genes. Clin Genet. 2017;92(4):415-22.

Duat Rodriguez A, Prochazkova M, Santos Santos S, Rubio Cabezas O, Cantarin Extremera V, Gonzalez-Gutierrez-Solana L. Early Diagnosis of CAPOS Syndrome Before Acute-Onset Ataxia-Review of the Literature and a New Family. Pediatr Neurol. 2017;71:60-4.

ISCN 2016 : An International System for Human Cytogenomic Nomenclature; McGowan-Jordan J, Simons A, Schmid M (eds). Cytogenet Genome Res. 2016;149:1-140.

Kessi M, Xiong J, Wu L, Yang L, He F, Chen C, et al. Rare Copy Number Variations and Predictors in Children With Intellectual Disability and Epilepsy. Front Neurol. 2018;9:947.

Leonard H, Wen X. The epidemiology of mental retardation: Challenges and opportunities in the new millennium. Ment Retard Dev Disabil Res Rev. 2002;8(3):117-34.

Miller DT, Adam MP, Aradhya S, Biesecker LG, Brothman AR, Carter NP, et al. Consensus Statement: Chromosomal Microarray Is a First-Tier Clinical Diagnostic Test for Individuals with Developmental Disabilities or Congenital Anomalies. Am J Hum Genet. 2010;86(5):749-64.

Riggs ER, Andersen EF, Cherry AM, Kantarci S, Kearney H, Patel A, et al. Technical standards for the interpretation and reporting of constitutional copy-number variants: a joint consensus recommendation of the American College of Medical Genetics and Genomics (ACMG) and the Clinical Genome Resource (ClinGen). Genet Med. 2020;22(2):245-57.
Rodacker V, Toustrup-Jensen M, Vilsen B. Mutations Phe785Leu and Thr618Met in $\mathrm{Na}+, \mathrm{K}+-$ ATPase, associated with familial rapid-onset dystonia parkinsonism, interfere with $\mathrm{Na}+$ interaction by distinct mechanisms. J Biol Chem. 2006;281(27):18539-48.

Rosewich H, Ohlenbusch A, Huppke P, Schlotawa L, Baethmann M, Carrilho I, et al. The expanding clinical and genetic spectrum of ATP1A3-related disorders. Neurology. 2014; 82(11):945-55.

Roubergue A, Roze E, Vuillaumier-Barrot S, Fontenille MJ, Méneret A, Vidailhet M, et al. The multiple faces of the ATP1A3-related dystonic movement disorder. Mov Disord. 2013; 28(10):1457-9.

Sagoo GS, Butterworth AS, Sanderson S, ShawSmith C, Higgins JP, Burton H. Array CGH in patients with learning disability (mental retardation) and congenital anomalies: Updated systematic review and meta-analysis of 19 studies and 13,926 subjects. Genet Med. 2009; 11(3):139-46.

Schalock RL, Brown I, Brown R, Cummins RA, Felce D, Matikka L, et al. Conceptualization, measurement, and application of quality of life for persons with intellectual disabilities: Report of an international panel of experts. Ment Retard. 2002;40(6):457-70.

Zablotsky B, Black LI, Maenner MJ, Schieve LA, Danielson ML, Bitsko RH, et al. Prevalence and trends of developmental disabilities among children in the United States: 20092017. Pediatrics. 2019;144(4):e20190811. 